\title{
Electronic and Magnetic Properties of Double Perovskites $\mathrm{Nd}_{2} \mathrm{MgIrO}_{6}$
}

\author{
Madhav Prasad Ghimire ${ }^{1^{*}}$, Gopi Chandra Kaphle ${ }^{1,2}$ and R. K. Thapa ${ }^{1,3}$ \\ ${ }^{1}$ Condensed Matter Physics Research Center, Butwal, Rupandehi, Nepal \\ ${ }^{2}$ Department of Physics, Tri-Chandra Multiple Campus, Ghantaghar, Kathmandu, Nepal \\ ${ }^{3}$ Department of Physics, Mizoram University, Aizawl 796009, Mizoram, India \\ "Correspondence to: ghimire.mpg@gmail.com
}

\begin{abstract}
We have studied the electronic and magnetic properties of double perovskites $\mathrm{Nd}_{2} \mathrm{MgIrO}_{6}$ by means of full-potential linearized augmented plane wave (FP-LAPW) method based on density-functional theory (DFT). For the exchange-correlation potential, generalized gradient approximation (GGA) has been used. Based on our DFT calculations, $\mathrm{Nd}_{2} \mathrm{MgIrO}_{6}$ is found to have an antiferromagnetic (AFM) ground state. The material shows Mott-Hubbard type insulator, which is observed to occur due to strong correlation in Nd-4f and Ir-5d states in addition to large crystal distortion, observed in the system. Strong hybridization between $O-2 p, I r-5 d$ and $N d-4 f$ electrons are observed from the density of states findings. Our results shows that the $5 d$ electrons of Ir hybridize strongly with $O$-2p states close to the Fermi level giving rise to the insulating state with a Mott-gap of $\sim 0.9 \mathrm{eV}$ in $\mathrm{Nd}_{2} \mathrm{MgIrO}_{6}$. Our study suggests that the total magnetic moment reduces to $5.0 \mu_{B}$ per formula unit as a result of itinerant super-exchange rather than the exchange interaction involving individual ions of Nd and Ir atoms.
\end{abstract}

Keywords: First-principles calculations, antiferromagnetism, Mott-Hubbard insulator.

PACS: 71.15.Mb; 71.27.+a; 71.20.Eh; 75.50.Cc; 71.30.+h.

\section{Introduction}

Perovskite oxides, a broad class of compounds with $\mathrm{ABO}_{3}$ structure are one of the most studied families of compounds in recent years owing greatly to their synthetic variability, compositional flexibility and their intriguing properties apart from their applications in electronic and magnetic devices. The general chemical formula of double perovskites can be expressed as $\mathrm{A}_{2} \mathrm{~B}^{\prime} \mathrm{B}^{\prime \prime} \mathrm{O}_{6}$, where $\mathrm{A}$ is an alkaline earth such as $\mathrm{Nd}, \mathrm{Sr}$, or $\mathrm{Ba}$, etc. and $\mathrm{B}^{\prime} \mathrm{B}^{\prime \prime}$ sites are occupied alternately by different transition metals, MgIr, LiIr, FeMo, FeRe, MnMo, etc. [Sleight and Ward, 1961; Longo and Ward,
1961; Mitchel, 2002]. Perovskites oxides have been studied since 1950 when ferromagnetic behavior is observed in manganites $\left(\mathrm{AMnO}_{3}\right.$; $\mathrm{A}=$ divalent or trivalent cations) around room temperature [Jonker and van Santen, 1950]. In these compounds, the existence of mixed valence in Mn allowing for an electron transfer through oxygen orbitals was invoked in order to explain the ferromagnetic behaviour via a double exchange mechanism [Zener, 1951]. Observation of half-metallic ferromagnetism (HMF) triggered a new interest in these type of compounds [Ghimire et al., 2015] when 
considerable magneto-resistance behavior and a high magnetic transition temperature which are important for the operation of devices at room temperatures [Kobayashi et al., 1998]. The HMF's has potential applications in the field of spin electronics due to their important properties like half-metallicity, high magnetic ordering temperature and magneto-dielectricity [Ghimire et al., 2011]. In these classes of compounds, the super exchange interactions between magnetic ions are in the form of $\mathrm{B}^{\prime}-\mathrm{O}-\mathrm{B}^{\prime \prime}$, instead of $\mathrm{B}-\mathrm{O}-\mathrm{B}$ form in simple perovskites of $\mathrm{ABO}_{3}$.

Experimentally there had been a number of studies on crystal growth, crystal structure and magnetic properties of $5 \mathrm{~d}$ double perovskites oxides. Glasso and Darby [1965] reported the existence of the compounds $\mathrm{La}_{2} \mathrm{MIrO}_{6}$ for $\mathrm{M}=\mathrm{Mg}, \mathrm{Mn}$ and Ni while Blasse [1965] reported the occurrence of the isostructural compounds for $\mathrm{M}=\mathrm{Mg}, \mathrm{Co}, \mathrm{Ni}$ and $\mathrm{Cu}$. Currie et al. [1995] also studied the structure and magnetic properties of $\mathrm{La}_{2} \mathrm{MIrO}_{6}(\mathrm{M}=\mathrm{Mg}$, $\mathrm{Co}, \mathrm{Ni}$ and $\mathrm{Zn}$ ). The magnetic susceptibility measurement shows magnetic ordering for $\mathrm{M}=\mathrm{Co}$ and Ni. Likewise, Mugavero et al. [2010] studied a series of lanthanide oxides $\mathrm{Ln}_{2} \mathrm{MgIrO}_{6}$ (where $\mathrm{Ln}=\mathrm{Pr}, \mathrm{Nd}, \mathrm{Sm}-\mathrm{Gd}$ ) and determine the ferromagnetic and anti-ferromagnetic behavior. Recent study by Yahua, et al. [2015] shed more attractive features on high-pressures synthesis, crystal structures and magnetic properties of osmium based double-perovskites.

However, due to the complicated characteristics of lanthanide oxides, there are limited theoretical works carried out on these systems to determine their properties. Hence, in the present work efforts have been taken to study the electronic and magnetic properties of double perovskites $\mathrm{Nd}_{2} \mathrm{MgIrO}_{6}$ by first principles density functional approach. A newly synthesized double perovskite $\mathrm{Nd}_{2} \mathrm{MgIrO}_{6}$ (NMIO) from the family of iridates is of particular interest [Ghimire et al., 2014; Ghimire et al., 2015; Pesin and Balents, 2010] due to its unique properties: (i) A-site element $\mathrm{Nd}$ and B'-site element Ir provides charge as well as spin, and (ii) topmost occupied states close to Fermi level $\left(E_{\mathrm{F}}\right)$ are exclusively spindown bands contributed by $d$ electrons of Ir atoms, and (iii) $\mathrm{IrO}_{6}$ octahedron exhibit large crystal distortion which may induce strong crystal field that helps in splitting the spin-up and spin-down bands near $E_{\mathrm{F}}$.

\section{Crystal Structure and Computational Details}

$\mathrm{Nd}_{2} \mathrm{MgIrO}_{6}$ crystallize in the space group $P 2_{1} / \mathrm{n}$ with the monoclinic-distorted double perovskites structure as shown in Fig. 1. The titled compound has structural distortions due to the tilting and rotation of $\mathrm{IrO}_{6}$ octahedron in addition to the different bond lengths between Ir and oxygen atoms.

The electronic and magnetic properties of $\mathrm{Nd}_{2} \mathrm{MgIrO}_{6}$ are studied by using full-potential linearized augmented plane wave (FP-LAPW) method based on density functional theory(DFT) as implemented in the WIEN2k code [Blaha et al., 2001]. The package is a direct application of full-potential and thus is considered as one of the most accurate computational methods. In the FP-LAPW method, the solution to the Kohn Sham equations [Kohn and Sham, 1965] are performed self-consistently, and the augmented plane wave plus local orbital basis set is incorporated to represent the electronic band structure for all atoms and their corresponding orbitals. Here, the core states are treated fully relativistically while the semi-core and valence 


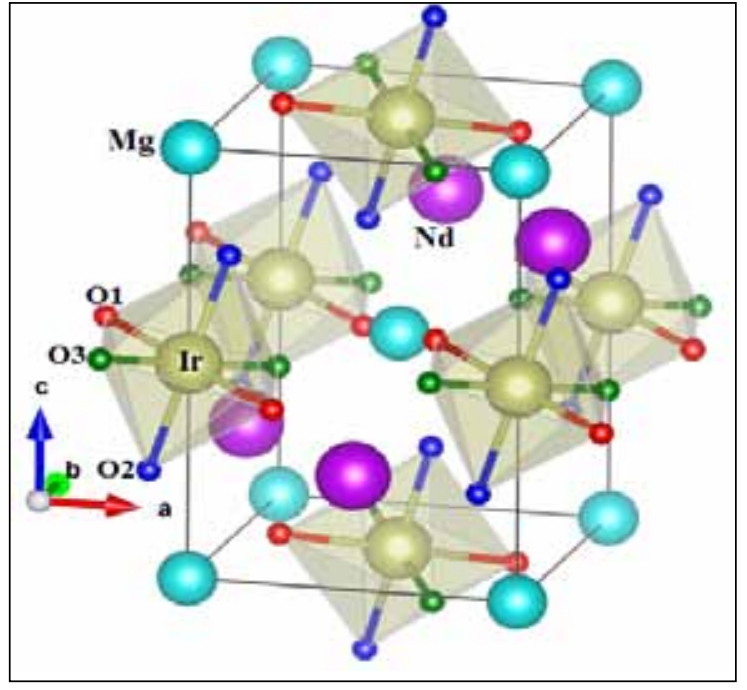

Figure 1. Crystal structure of the double perovskite $\mathrm{Nd}_{2} \mathrm{MgIrO}_{6}$. Distortion due to tilting and rotation of $\mathrm{IrO}_{6}$ octahedron are shown.

states are treated semi-relativistically. The standard generalized-gradient approximation (GGA) exchange correlation potential within the PBE-scheme were used with Coulomb interaction U [Perdew et al., 1996; Anisimov et al., 1997]. The results shown here is with $\mathrm{U}_{\mathrm{Nd}}=6$ $\mathrm{eV}$ and $\mathrm{U}_{\mathrm{Ir}}=1.25 \mathrm{eV}$ respectively [Ghimire et al., 2014]. We have chosen the muffin-tin radii for $\mathrm{Nd}, \mathrm{Mg}, \mathrm{Ir}, \mathrm{O} 1, \mathrm{O} 2$ and $\mathrm{O} 3$ to be 2.34, 1.92, $1.99,1.76,1.76$ and 1.77 a.u. respectively. Integrations in reciprocal space were performed using 108 spatial $k$-points in the irreducible wedge of the Brillouin zone.

\section{Results and Discussions}

$\mathrm{Nd}_{2} \mathrm{MgIrO}_{6}$ belongs to double perovskite where $\mathrm{Nd}$ at A-site provides charge to the system and nominally take the charge state +3 with $4 \mathrm{f}^{3}$ configuration, lying deep in the valence region below the Fermi level $\left(E_{\mathrm{F}}\right)$. Unlike other cases however, $\mathrm{Nd}$ is in a high-spin state due to strong Hund's coupling. The transition element
Ir nominally takes the charge state +4 with $5 d^{5}$ configuration where five of the totally six $t_{2 g}$ orbits are occupied and lie at the top of the valence band, forming a low-spin state due to large crystal field from oxygen octahedron.

From first-principles DFT calculations, we observed that the $\mathrm{Nd}$ couples antiferromagnetically with Ir giving rise to antiferromagnetic ground state consistent with the experimental prediction [Mugavero et $a l ., 2010]$. According to the calculations, the material NMIO shows an insulating state (see Fig. 2). Since Nd and Ir are strongly correlated, $\mathrm{GGA}+\mathrm{U}$ was considered wherein the material is found to be a Mott-Hubbard insulator with an energy gap of approx. $0.9 \mathrm{eV}$ at EF (see Fig. 2 and Fig. 3). From the total and partial (Nd4f, Ir-5d, O-2p) DOS shown in Fig. 2 and the band structure in Fig. 3, it is observed that the occupied $\mathrm{Nd}-4 f$ states lie deep in the valence region for spin-up channel whereas for spindown, they appear far from $E_{\mathrm{F}}$ in the conduction region. This gives rise to large exchange energy splitting $(\sim 3 \mathrm{eV})$ in $\mathrm{Nd}$. The Ir-5d states are found to play key role in dictating the electronic properties of NMIO. They are found to hybridize strongly with the O-2p states in both spin channels. Their hybridization occurs mostly in the valence region near $E_{\mathrm{F}}$ and in the conduction region. We observe that $\operatorname{Ir}_{-t_{2 g}}$ (i. e., $\mathrm{d}_{\mathrm{xy}}, \mathrm{d}_{\mathrm{xz}}$, and $\mathrm{d}_{\mathrm{yz}}$ ) states are fully occupied in spin-down channels and thus lies in the valence region whereas in spin-up channel, only two out of three $t_{2 g}$ states (i. e., $d_{x y}, d_{x z}$ ) are occupied while the remaining $\mathrm{d}_{\mathrm{yz}}$ states being empty lies in the conduction region along with the other un-occupied Ir-de ${ }_{g}$ states. This picture is found consistent with the ionic picture. Charge transfer effect is prominent between Ir-5d and $\mathrm{O}-2 \mathrm{p}$ states due to strong hybridization. This 


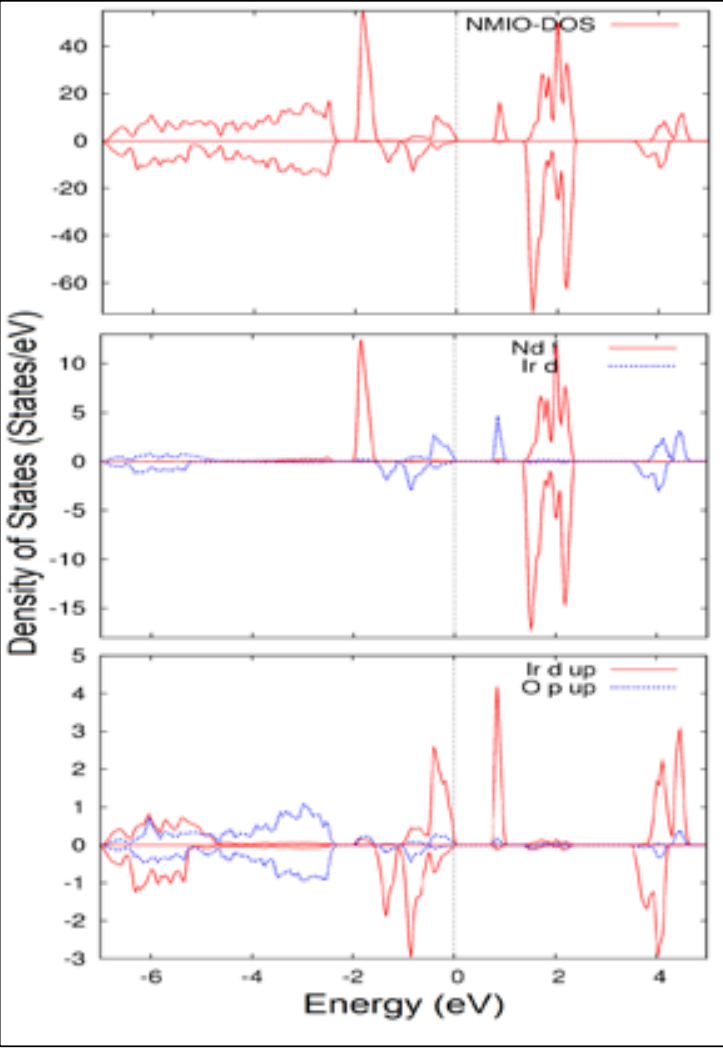

Figure 2. Total and partial density of states GGA+U for $\mathrm{Nd}_{2} \mathrm{MgIrO}_{6}$ : $\mathrm{Nd}-4 \mathrm{f}, \mathrm{Ir}-5 \mathrm{~d}$ and $\mathrm{O}-2 \mathrm{p}$ respectively.

induces sizable moment in oxygen atoms which get polarized in parallel with the Ir atoms. We observe that there is no exchange splitting between $\mathrm{O}-2 \mathrm{p}$ and $\mathrm{Mg}$-2sp (not shown) electrons while for Nd-4f and Ir-5d DOS, there is an exchange splitting between spin-up and spin-down configurations. Exchange splitting energy of Ir- $5 \mathrm{~d}$ and $\mathrm{Nd}-4 \mathrm{f}$ is found to be $\sim 2 \mathrm{eV}$ and $\sim 3 \mathrm{eV}$ respectively which contributes to antiferromagnetic coupling between $\mathrm{Nd}$ and $\mathrm{Ir}$ in $\mathrm{Nd}_{2} \mathrm{MgIrO}_{6}$.

From first-principles calculations, we obtain magnetic moment of $\mu \approx+2.87 \mu_{\mathrm{B}}$ per Nd atom, $\mu \approx-0.63 \mu_{\mathrm{B}}$ per Ir atom and $\mu \approx 0.1$ $\mu_{\mathrm{B}}$ per one set of three $\mathrm{O}$ atoms, respectively, with a total magnetic moment of $5.0 \mu_{\mathrm{B}}$ per

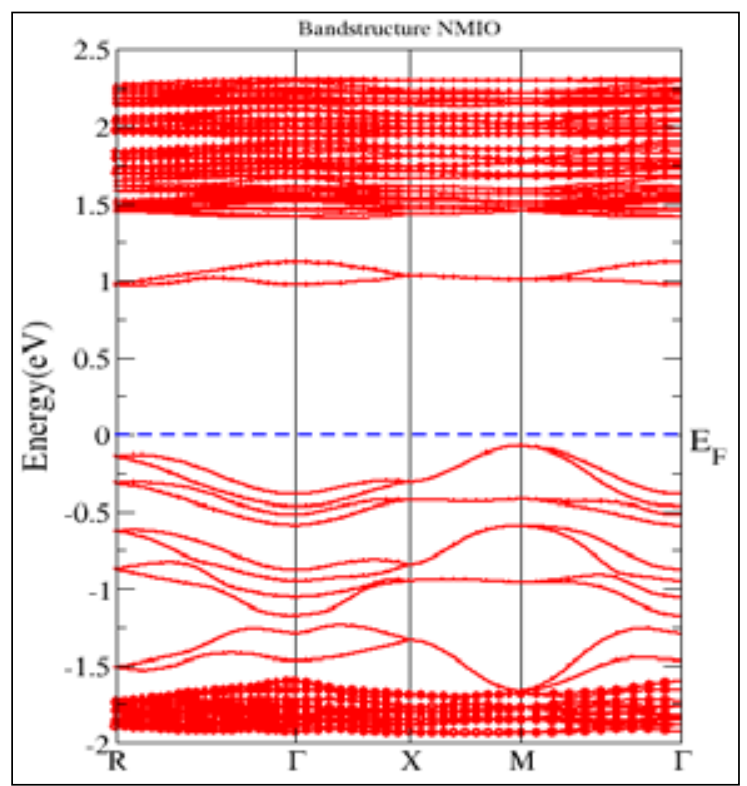

Figure 3. Band structures of $\mathrm{Nd}_{2} \mathrm{MgIrO}_{6}$ within $\mathrm{GGA}+\mathrm{U}$ scheme.

formula unit which is slightly larger than the experimental moment of $\mu_{\mathrm{tot}}=5.17 \mu_{\mathrm{B}}$ per formula unit. This discrepancy is expected to resolve when considering the spin-orbit coupling (work in progress) in $\mathrm{Nd}$ and Ir because both elements with less than half-filled shell will have their orbital moment anti-parallel to spin-moment which may further increase the total magnetic moment close to the experimental moment.

\section{Conclusions}

On the basis of first-principles density functional calculations, we propose $\mathrm{Nd}_{2} \mathrm{MgIrO}_{6}$ to be an antiferromagnetic Mott-Hubbard insulator. Our study suggests that the enhanced magnetic moment is a result of itinerant exchange rather than the exchange interaction involving individual ions of $\mathrm{Nd}$ and $\mathrm{Ir}$ atoms.

\section{Acknowledgments}

This work was supported partially by CMPRC, Butwal (Nepal) and the National plan for science, 
technology and innovation (India) under the research project No. 11-NAN1465-02. Editor N. P. Chapagain thanks the reviewers for their assistance in evaluating this paper.

\section{References}

Anisimov, V. I., Aryasetiawan, F. and Lichtenstein, A. I. (1997). First-principles calculations of the electronic structure and spectra of strongly correlated systems: the LDA+ U method. $J$. Phys.: Condens. Matter, 9, 767.

Blaha, P., Schwarz, K., Madsen, G. K. H., Kvasnicka, D. and Luitz, J. (2001). An augmented plane wave and local orbital program for calculating crystal properties. Karlheinz Schwarz, Tech. Universitat. Wien, Austria, ISBN 3-9501031-12.

Blasse, G. (1965). New compounds with perovskitelike structures. J. Inorg. Nucl. Chem., 27, 993.

Curie, R. C., Vente, J. F., Frikkee, E. and IJdo, D. J. W. (1995). The structure and magnetic properties of $\mathrm{La}_{2} \mathrm{MIrO}_{6}$ with $\mathrm{M}=\mathrm{Mg}, \mathrm{Co}, \mathrm{Ni}$, and $\mathrm{Zn}$. $J$. Sol. Stat. Chem., 116, 199-204.

Ghimire, M. P., Thapa, R. K., Rai, D. P., Sandeep, Sinha, T. P. and Hu, X. (2015). Half metallic ferromagnetism in tri-layered perovskites $\mathrm{Sr}_{4} \mathrm{~T}_{3} \mathrm{O}_{10}(\mathrm{~T}=\mathrm{Co}, \mathrm{Rh})$. J. Appl. Phys., 117, 063903 .

Ghimire, M. P., Sandeep, Sinha, .T. P. and Thapa, R. K. (2011). First principles study of the electronic and magnetic properties of semi-Heusler alloys $\mathrm{NiXSb}(\mathrm{X}=\mathrm{Ti}, \mathrm{V}, \mathrm{Cr}$ and $\mathrm{Mn})$. J. Alloys Compds. 509, 9742 .

Ghimire, M. P., Wu, L. H. and $\mathrm{Hu}, \mathrm{X}$. (2014). Half metal transition driven by doping effects in osmium double perovskite, eprint arXiv:1407.3408.

Ghimire, M. P., Wu, L. H. and Hu, X. (2015). Halfmetalic ferrimagnetism in hole doped lanthanide iridates. J. Supercond. Nov. Mag., 28, 917.

Glasso, F. and Darby, W. (1965). Preparation of single crystals of complex perovskite ferroelectric and semi conducting compounds. Inorg. Chem., 4, 71.
Jonker, G. H. and van Santen, J. H. (1950). Ferromagnetic compounds of manganese with perovskite structure, Physica, 16, 337 .

Kobayashi, K. I., Kimura, T., Terakura, H., and Tokura, Y. (1998). Room-temperature magnetoresistance in an oxide material with an ordered double-perovskite structure. Nature, 395, 677 .

Kohn, W. And Sham, L. J. (1965). Self-consistent equations including exchange and correlation effects. Phys. Rev., 140, A1133.

Longo, J. M. and Ward, R. (1961). Magnetic compounds of hexavalent rhenium with the perovskite-type structure. J. Am. Chem. Soc., 83, 2816.

Mitchell, R. M. (2002). Perovskites: Modern and ancient. Almaz Press, Thunder Bay, Ontario, ISBN-0968941109.

Mugavero III, S. J., Fox, A. H., Smith, M. D., and zur Loye, H.-C. (2010). Crystal growth, structure and magnetic properties of the double perovskites $\mathrm{Ln}_{2} \mathrm{MgIrO}_{6}(\mathrm{Ln}=\mathrm{Pr}, \mathrm{Nd}, \mathrm{Sm}-\mathrm{Gd}) . J$. Sol. Stat. Chem., 183, 465.

Perdew, J. P., Burke, K. and Ernzerhof, M. (1996). Generalized gradient approximation made simple. Phys. Rev. Lett., 77, 3865.

Pesin, D., and Balents, L. (2010). Mott physics and band topology in materials with strong spinorbit interaction. Nat. Phys., 6, 376.

Sleight, A. W. and Ward, R. (1961). Compounds of heptavalent rhenium with the perovskite structure. J. Am. Chem. Soc., 83, 1087.

Yahua, Y., Feng, H. L., Ghimire, M. P., Matsushita, Y., Tsujimoto, Y., He, J., Tanaka, M., Katsuya, Y. and Yamaura, K. (2015). High-pressure synthesis, crystal structures and magnetic properties of $5 \mathrm{~d}$ double-perovskite oxides $\mathrm{Ca}_{2} \mathrm{MgOsO}_{6}$ and $\mathrm{Sr}_{2} \mathrm{MgOsO}_{6}$. Inorg. Chem., 54, 3422.

Zener, C. (1951). Interaction between the d-Shells in the transition metals: II. Ferromagnetic compounds of manganese with perovskite structure. Phys. Rev., 82, 403. 